\title{
Atomic Resolution Vibrational Spectroscopy with On-Axis Detector Geometry
}

\author{
Kartik Venkatraman*1, Barnaby D.A. Levin ${ }^{1}$, Katia March $^{2}$, Peter Rez ${ }^{3}$ and Peter A. Crozier ${ }^{1}$ \\ 1. School for the Engineering of Matter, Transport and Energy, Arizona State University, Tempe, \\ Arizona, USA. \\ 2. Eyring Materials Center, Arizona State University, Tempe, Arizona, USA. \\ 3. Department of Physics, Arizona State University, Tempe, Arizona, USA. \\ * Corresponding author: kvenka16@asu.edu
}

Atomic-scale characterization of vibrational modes in materials can greatly improve our understanding of ionic and atomic diffusion, heat transport, phase transformations, and surface chemical reactions. The detection of vibrational modes with monochromated vibrational electron energy-loss spectroscopy (EELS) in a scanning transmission electron microscope (STEM) opens up the possibility to probe such excitations with high spatial resolution. The electron interactions associated with the excitation of vibrational modes can be discussed in terms of dipole and impact scattering as was done for highresolution electron energy-loss spectroscopy (HREELS) by Ibach and others [1]. Dipole scattering is associated with the long-range Coulomb field which excites vibrational modes by polarizing the medium while impact scattering is associated with short-range interactions with the nucleus and atomic electrons. Recent work on the influence of an abrupt $\mathrm{SiO}_{2} / \mathrm{Si}$ interface on the spatially resolved $\mathrm{Si}-\mathrm{O}$ bond stretch vibrational signal showed that the electron probe can sense the interface from a distance of $200 \mathrm{~nm}$ in $\mathrm{SiO}_{2}$ due to the dipolar character of the $\mathrm{Si}-\mathrm{O}$ vibrational mode [2]. This delocalization in the vibrational signal can be suppressed either by selectively probing chemical bonds with no ionic character or nondipole vibrational modes like acoustic phonons or symmetric-stretch modes in ionic materials. In this paper, we demonstrate that it is possible to achieve atomic spatial resolution with individual vibrational modes in $\mathrm{Si}$, which has completely covalent bonding character, and in $\mathrm{SiO}_{2}$, which has mixed ionic and covalent bonding character, in the conventional on-axis STEM EELS geometry.

A conventional cross section Si sample in the [110] zone axis orientation was prepared by dimpling and milling. A second sample was prepared for vibrational EELS experiments across the $\mathrm{SiO}_{2} / \mathrm{Si}$ interface by lifting out a FIB lamella from an oxidized Si wafer. STEM-EELS analysis on all samples was performed using a NION UltraSTEM 100 aberration-corrected electron microscope equipped with a monochromator, operated at $60 \mathrm{kV}$. The probe convergence semi-angle was $28 \mathrm{mrad}$, and a $1 \mathrm{~mm}$ spectrometer entrance aperture was used corresponding to a collection semi-angle of $12 \mathrm{mrad}$. A dispersion of 1 or $2 \mathrm{meV}$ per channel was used to record all spectra. Aberration correction of the magnetic lenses up to the fifth order produced probes of $\sim 0.12 \mathrm{~nm}$ diameter with beam currents of $\sim 100$ $\mathrm{pA}$. During the monochromated experiment, the beam current was $\sim 10 \mathrm{pA}$, and the energy resolution was $15 \mathrm{meV}$.

Fig. 1a shows the conventional on-axis spectrometer geometry for EELS in the STEM employed in this work. Fig. 1b shows a monochromated ADF image of the Si dumbbells and the position of the EELS linescan. The monochromated probe size was $\sim 0.17 \mathrm{~nm}$, which wasn't small enough to resolve the $\mathrm{Si}$ atoms forming the dumbbell. Fig. 1c shows the background subtracted vibrational energy-loss spectra at every $0.06 \mathrm{~nm}$ between the points marked in the Fig. 1b. There are very significant differences in the spectral shape and intensity as the probe changes position. The spectrum shows two peaks when the probe is between dumbbell columns; one at $62 \pm 2 \mathrm{meV}$ with a width of approximately $16 \mathrm{meV}$ and a 
weaker broader feature centered at about $43 \pm 2 \mathrm{meV}$. The higher energy peak corresponds to optical phonons near the center of the Brillouin zone whereas the lower energy peak corresponds to optical and acoustic phonons at the Brillouin zone boundary, as can be ascertained from the blue (high-energy) and red (low-energy) bands in the Si phonon dispersion curves, shown in Fig. 1d. When the probe is on the column (position 4), only one peak is apparent with energy about $58 \mathrm{meV}$ and width of $22 \mathrm{meV}$. As the probe continues to move off the column, it becomes narrower and shifts back to approximately $62 \mathrm{meV}$ (positions 5 and 6). The integrated intensity of the peak on the column is approximately 3 times the intensity between the columns and shows a spatial resolution of better than $0.2 \mathrm{~nm}$ for both the high and low energy peaks. Experimental observations from $\mathrm{Si}$ and $\mathrm{SiO}_{2}$ will be discussed in more detail [4].

\section{References:}

[1] H Ibach and D.L. Mills in "Electron Energy Loss Spectroscopy and Surface Vibrations (Academic Press).

[2] K Venkatraman et al., Microscopy 67 (2018), p. i14.

[3] SP Ong et al., Computational Materials Science 68 (2013), p. 314.

[4] The support from National Science Foundation CHE-1508667 and the use of (S)TEM at Eyring Materials Center at Arizona State University is gratefully acknowledged.
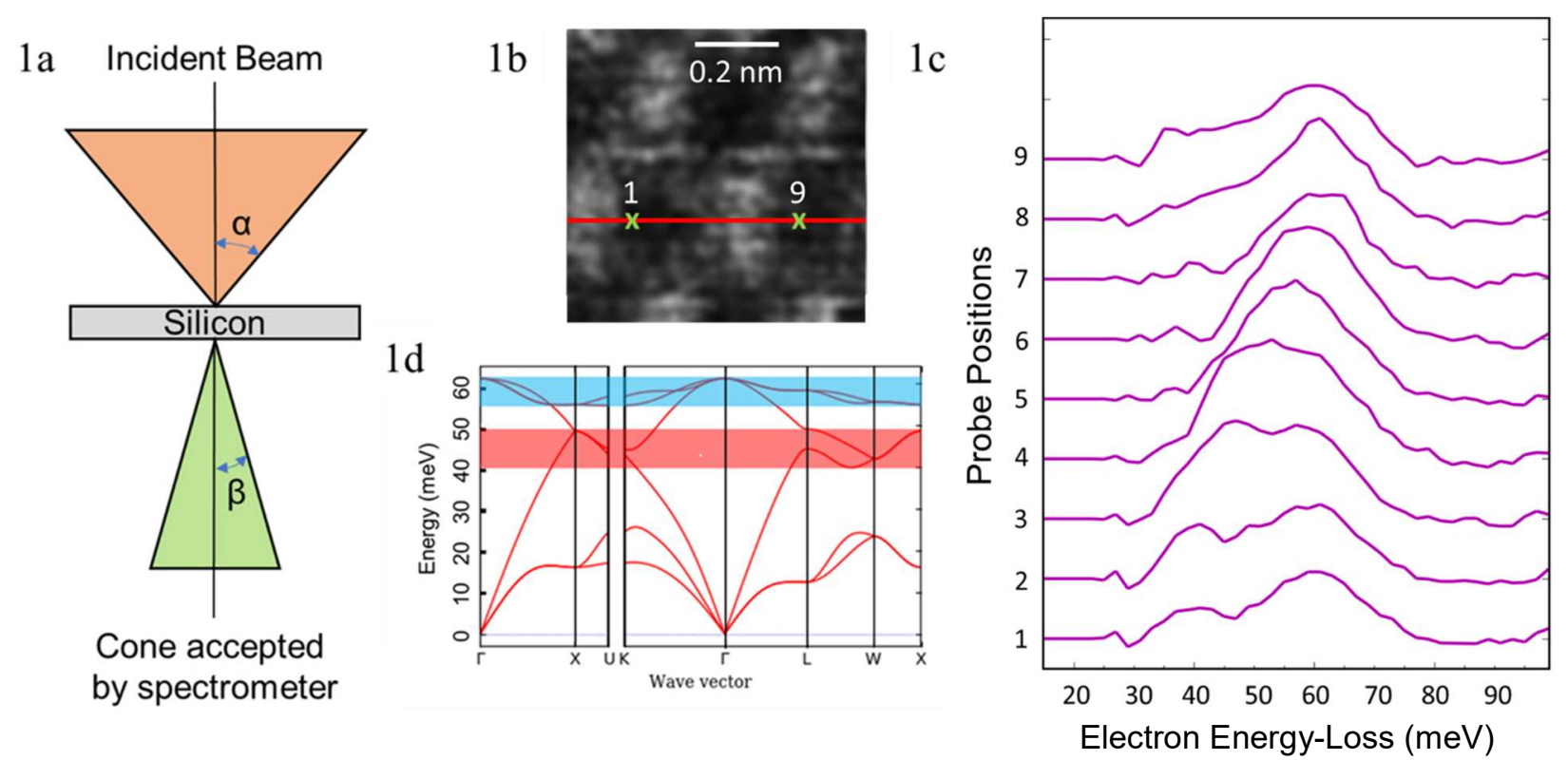

Figure 1. (a) Schematic diagram showing the STEM EELS acquisition geometry used in experiment with $\alpha=28 \mathrm{mrad}$ and $\beta=12 \mathrm{mrad}$. (b) ADF image of Si dumbbells with the red line indicating position of EELS linescan acquisition. (c) Raw spectra at 9 individual probe positions all separated by $0.06 \mathrm{~nm}$ along the linescan between the labels 1 and 9 shown in b. (d) Dispersion surfaces in for $\mathrm{Si}$ - blue and red shaded areas corresponds to peaks in the density of states on the upper branches from $55-62 \mathrm{meV}$ (optical) and on a lower branch from $41-48 \mathrm{meV}$ (optical and acoustic) [3]. 